\title{
Bound for Specific Sounds: Vocal Predisposition in Animal Communication
}

Garcia, Maxime ; Manser, Marta

\begin{abstract}
Mechanical constraints imposed by anatomical adaptations are a ubiquitous feature of animal sound production. They can give rise to 'vocal predispositions' (i.e., acoustic structures strictly determined by vocal anatomy). Such predispositions are crucial to the investigation of the cognitive and evolutionary processes underlying acoustic communication in vertebrates, including human speech.
\end{abstract}

DOI: https://doi.org/10.1016/j.tics.2020.05.013

Posted at the Zurich Open Repository and Archive, University of Zurich

ZORA URL: https://doi.org/10.5167/uzh-190746

Journal Article

Accepted Version

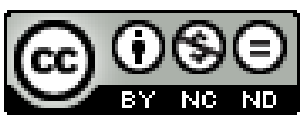

The following work is licensed under a Creative Commons: Attribution-NonCommercial-NoDerivatives 4.0 International (CC BY-NC-ND 4.0) License.

Originally published at:

Garcia, Maxime; Manser, Marta (2020). Bound for Specific Sounds: Vocal Predisposition in Animal Communication. Trends in Cognitive Sciences, 24(9):690-693.

DOI: https://doi.org/10.1016/j.tics.2020.05.013 
Q2

3

6

Q4 Q3 Maxime Garcia ${ }^{1, *}$ and

8

9

10 Mechanical constraints imposed 11 by anatomical adaptations are a 12 ubiquitous feature of animal sound 13 production. They can give rise to 14 'vocal predispositions' (i.e., acoustic 15 structures strictly determined by 16 vocal anatomy). Such predisposi17 tions are crucial to the investigation 18 of the cognitive and evolutionary 19 processes underlying acoustic com20 munication in vertebrates, including 21 human speech. cognitive control over sound production? We address this question in light of the most recent breakthroughs in the fields of bioacoustics and comparative linguistics [additional references are listed online (see the supplementary information online)]. We define 'vocal predispositions' as a range of acoustic features predetermined by species-specific vocal anatomy. We emphasize why identifying vocal predispositions is essential to infer evolutionary histories and the cognitive processes underlying the structure and use of acoustic signals.

Anatomical Adaptations Generate Vocal Predispositions

Acoustic communication has evolved as a behavioral keystone in most of the animal taxa. Across vertebrates, sound-generating organs come in diverse forms and are often characteristic of the clade considered: Typically, birds produce calls with a syrinx, while mammals and amphibians do so using their larynx, and fish their swim bladder [5]. However, deviations from typical structures occur in each of these clades to varying degrees as a result of various selective forces. Modifications of the vocal apparatus can, for instance, originate from sexual selection, as seen in the context of dishonest signalling: Here, changes in vocal anatomy result from an attempt to exaggerate the conveyed impression of body size [1]. Environmental factors can also selectively operate on acoustic features by triggering adaptive changes in a species' vocal anatomy. For instance, there is suggestive evidence that some terrestrial vertebrates have evolved air sacs as an impedance-matching system for sounds to better radiate into their surrounding medium, a function similar to that of phonic lips and melons in several aquatic mammals [5].

Such selection pressures have led acoustic signals to inherently contain a range of features that may not be physically reproducible by other species (e.g., the low fundamental frequencies produced by howler monkeys or the low formant frequency spacing achieved by red deer stags [1]). In 47 other words, vocal predispositions emerge 48 from the biomechanical properties of the 49 sound-generating apparatus available to 50 each species. While this seems obvious, it 51 represents a critical - but often neglected - 52 basis for frameworks investigating the 53 various levels of neural control shaping 54 acoustic signals [6].

\section{Sound Production with or Without 56 a Brain 57} Understanding how vocal anatomy physi- 58 cally bounds sound production can strongly 59 affect our interpretation of the cognitive 60 underpinnings of acoustic communication 61 systems. The biomechanical properties of 62 sound production organs can generate 63 complex acoustic patterns despite simple 64 input conditions devoid of neural control. 65 For instance, naturally occurring vocal 66 sequences in squirrel monkeys can result 67 from nonlinearities (register breaks and 68 rapid transitions between acoustic patterns 69 or call types) that are intrinsic to laryngeal 70 biomechanics in this species [7]. In this 71 example, vocal sequence generation thus 72 results from vocal predispositions and 73 does not require advanced neural control. 74 Experimental designs aimed at phonating 75 excised vocal apparatuses ex vivo (hereafter 76 'EVAE' for 'excised vocal apparatus ex- 77 perimentation') provide an ideal tool to 78 investigate this topic. Free from any neural 79 control, this type of approach provides a 80 biomechanical baseline on which to build in- 81 ferences about the cognitive processes as- 82 sociated with vocal motor control (Figure 1). 83

As part of the research exploring these cog- 84 nitive capacities supporting communication, 85 vocal production learning (VPL; referred to 86 as the ability to imitate or modify existing 87 vocalizations to produce noninnate vocal 88 signals) has received particular attention. 89 VPL requires advanced motor control over 90 vocal organs [8] in order to produce the 91 spectral and/or temporal modulations typi- 92 cal of vocally learned signals (e.g., [9]). In 93 vertebrates, VPL is found in many birds 94 


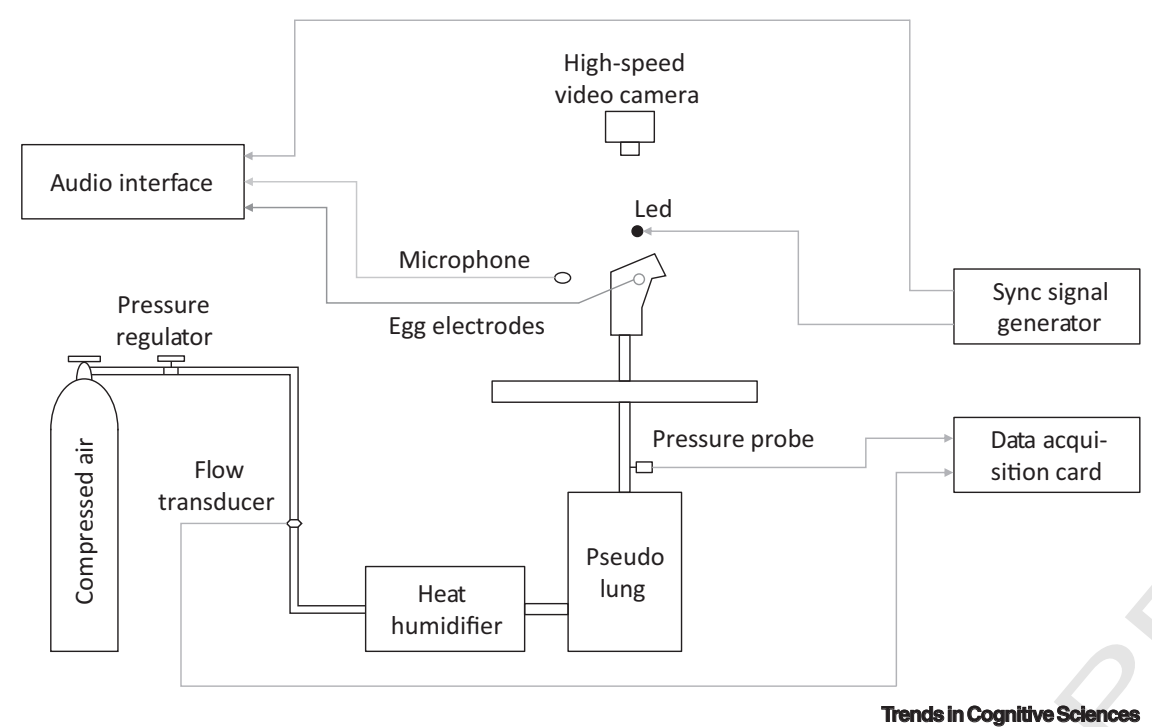

Q1 setup (adapted from [12]). Excised larynx experimentation (ELE; similar to excised syrinx experimentation in birds) consists of phonating a larynx ex vivo by driving pressurized air through it. ELE allows investigating sound production mechanisms while parceling out neural control. Combined with dissections, this approach reveals the biophysical behaviour of species-specific larynges and thus provides direct insight into vocal predispositions. As an example, ELE is a highly promising tool to disentangle the origin of nonlinear dynamics (NLD) occurring in animal sound production. NLD depend on a combination of input parameters (such as subglottal pressure and vocal fold tension), but not all larynges behave similarly with regard to NLD production. Research in this area can help improve understanding of whether NLD are evolutionary by-products or functional adaptations, which is essential to quantify vocal repertoires and determine the degree of volitional control on acoustic structure and use of vocalizations. Extending excised experimentation to the upper vocal tract will strongly complement computational modelling of dynamic filter-related vocal features and is a promising frontier that bioacousticians should tackle in the future. Abbreviations: LED, light emitting diode.

and few mammalian clades (including humans), and its assessment commonly relies on comparative approaches. Anatomic constraints are a major component determining the outcome and interpretations of comparative VPL research, as illustrated by theoretically comparing humans and lyrebirds, two species with advanced VPL abilities. Humans are unlikely to rival lyrebirds that mimic sounds of chainsaws, camera shutters, or other bird species' songs (https://www.youtube.com/watch? $\mathrm{v}=$ $\mathrm{mSB} 71 \mathrm{jNq}-\mathrm{yQ}$ ); yet, one should not conclude that humans are not vocal learners, but rather that neural pathways [9] and vocal organs significantly differ in these clades. The same consideration of vocal motor control being contingent on vocal predispositions should always apply to avoid downplaying or inflating cognitive performances associated with VPL when comparing different taxa. In line with this, the use of stimuli matching the range of physical capabilities of species-specific vocal apparatus should be paramount in VPL research (Figure 2). This will be possible only through careful evaluation of vocal predispositions and considering VPL as a continuum [4].

A flagship study field illustrating advances made by considering vocal predispositions is that of language evolution, as highlighted by recent work showing that human speech can be shaped by biomechanical adjustments to dietary practices, such as bite configuration [10]. Comparative work also showed that the anatomic constraints imposed on monkeys' vocal tract were not responsible for the lack of speech emergence in our close relatives. 115 Instead, the dual consideration of vocal 116 anatomy and neural control provides com- 117 pelling evidence that the missing element is 118 a speech-ready brain to control their vocal 119 tract [2]. Similarly, a study controlled for 120 sound production biomechanics to demon- 121 strate that marmosets' brain circuitry con- 122 tributes to vocal development in this 123 species, as with the mechanism in place 124 for vocal learning emergence in humans 125 [3]. Finally, contrasting the mechanisms 126 responsible for speech production with 127 those involved in the production of non- 128 verbal vocal communication has been 129 suggested as a promising research avenue, 130 as it will help improve understanding of 131 volitional vocal control in humans [11]. 132 These integrative approaches illustrate how 133 much benefit can result from considering 134 anatomic constraints in the study of our 135 own language, including when developing 136 new testable frameworks [6].

137

Vocal Predispositions Towards a 138 Predictive Framework 139 Although the general shape of sound pro- 140 duction structures has remained relatively 141 conserved within clades, vocal adapta- 142 tions in response to natural and sexual 143 selections are widespread. Furthering our 144 understanding of vocal predispositions 145 (i.e., the acoustic signals' properties that 146 derive from interspecific anatomical varia- 147 tion) has potential to open new pathways 148 for animal vocal communication research. 149 In particular, we argue that future research 150 should focus on identifying the range of - 151 and, even more relevant, the differences 152 between - expected and observed vocali- 153 zations, given species-specific vocal anat- 154 omies. This approach can advance our 155 understanding of unique vocal features 156 by helping to identify the anatomic corre- 157 lates of species-specific vocal traits. Build- 158 ing on predictions (via vocal anatomy 159 modelling, EVAE, and knowledge from re- 160 lated species) while conducting cross- 161 species comparisons, deviations from 162 the norm (observed $=$ expected) can 163 


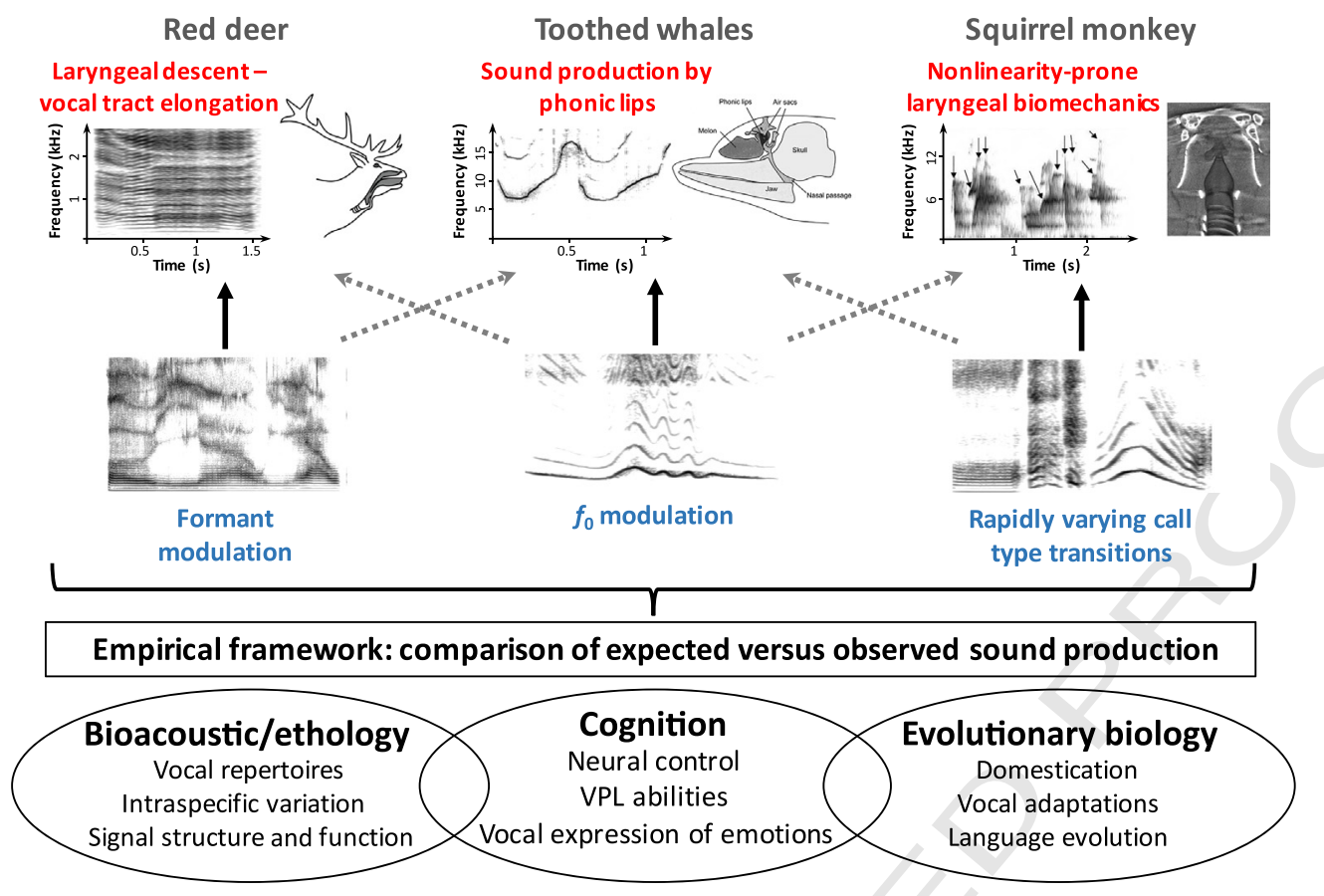

Squirrel monkey laryngeal biomechanics

Laryngeal descent -

vocal tract elongation $M$
Forman

modulation
(1) 3at

pidly varying call type transitions
(A)

Species-specific vocal predispositions

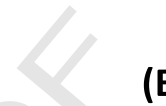

Stimulus categories to test vocal control abilities

Figure 2. Importance of Anatomic Constraints in Vocal Communication Research. (A) Anatomic adaptations and sound spectrograms highlighting vocal predispositions (in red) in three exemplar species' communication systems. For each species considered, acoustic signal structure is predetermined by vocal anatomy; we thus find formant lowering in red deer calls as they elongate their vocal tract, highly tonal signature whistle produced through phonic lips in toothed whales, and abrupt frequency jumps in a call sequence of squirrel monkeys, inherent to their laryngeal propensity for such jumps. Red deer illustration: courtesy of Tecumseh Fitch; toothed whale illustration: adapted from [5] and courtesy of Livio Favaro (audio available as supplementary material; see supplementary information online); squirrel monkey illustration: adapted with permission from [7], with arrows indicating successive elements in a vocal bout. (B) Hypothetical study testing vocal production learning (VPL) abilities in different species: This requires choosing appropriate stimuli (in blue) (i.e., stimuli tuned to the tested species' vocal predispositions). Naturally occurring vocalizations should help select or design biologically relevant experimental stimuli [formant modulation in red deer; fundamental frequency $\left(f_{0}\right)$ modulation in toothed whales; and nonlinear phenomena (changes in vocal fold vibratory regimes) in squirrel monkeys]. Solid arrows indicate the preferred stimulus to choose, given a model species' vocal predispositions. Dashed arrows indicate possible - but less appropriate, as potentially less in line with the model species' vocal predispositions - alternative stimuli. (C) Research areas that will benefit from adequate consideration of the vocal predispositions found across species.

potentially indicate restrained behavioral use of vocalizations (observed $<$ expected) or greater than average vocal motor control (observed $>$ expected). Reflections on (and empirical testing of the mechanisms responsible for such expected/observed differences can therefore provide critical insights into the volitional versus uncontrolled origin of acoustic signals and the relative influence of the selective pressures driving the evolution of acoustic communication systems.

\section{Acknowledgments}

The authors thank Dr A. Ravignani, Editor L. Drayton, and three anonymous reviewers for insightful comments on earlier versions of the manuscript.

\section{Supplementary Information}

Supplemental information associated with this article can be found online at https://doi.org/10.1016/j.tics. 2020.05.013.

${ }^{1}$ Animal Behaviour, Department of Evolutionary Biology and Environmental Studies, University of Zurich, Winterthurerstrasse 190, 8051, Zurich, Switzerland

${ }^{*}$ Correspondence:

maxime.garcia@ymail.com (M. Garcia).

https://doi.org/10.1016/j.tics.2020.05.013

References

1. Charlton, B.D. and Reby, D. (2016) The evolution of acoustic size exaggeration in terrestrial mammals. Nat. Commun. 7, 12739
2. Fitch, W.T. et al. (2016) Monkey vocal tracts are speech- 180 ready. Sci. Adv. 2, e1600723 181

3. Takahashi, D.Y. et al. (2015) The developmental 182 dynamics of marmoset monkey vocal production. Science 183 349, 734

4. Lattenkamp, E.Z. and Vernes, S.C. (2018) Vocal 185 learning: a language-relevant trait in need of a broad 186 cross-species approach. Curr. Opin. Behav. Sci. 21, 187 209-215 188

5. Ladich, F. and Winkler, H. (2017) Acoustic communication 189 in terrestrial and aquatic vertebrates. J. Exp. Biol. 220, 190 2306-2317 191

6. Zhang, Y.S. and Ghazanfar, A.A. (2020) A hierarchy of 192 autonomous systems for vocal production. Trends Neurosci. 193

5 43, 115-126 194

7. Brown, C.H. (2002) Ecological and physiological constraints 195 for primate vocal communication. In PrimateAudition: Ethology 196 and Neurobiology (Ghazanfar, A.A., ed.), pp. 127-150, 197 CRC Press 198

8. Stansbury, A.L. and Janik, V.M. (2019) Formant modifica- 199 tion through vocal production learning in gray seals. Curr. 200 Biol. 29, 2244-2249.e4 201

9. Tyack, P.L. (2020) A taxonomy for vocal learning. Philos. 202 Trans. R. Soc. Lond. B Biol. Sci. 375, $20180406 \quad 203$ 
204 10. Blasi, D.E. et al. (2019) Human sound systems are shaped 363 , eaav3218
11. Pisanski, K. et al. (2016) Voice modulation: A window into the origins of human vocal control? Trends Cogn. Sci. 20, 304-318
12. Herbst, C.T. et al. (2012) How low can you go? Physical 207 production mechanism of elephant infrasonic vocaliza- 208 tions. Science 337, 595-599 